\title{
Using digitized auditory stimuli on the Macintosh computer
}

\author{
JANET M. GIBSON \\ Rice University, Houston, Texas
}

\begin{abstract}
Hardware/software packages that digitize sound on the Apple Macintosh can help researchers prepare and present auditory materials needed for their experiments. Common features and benefits of commercially available sound digitizing packages are discussed in terms of some possible applications to cognitive psychology experiments.
\end{abstract}

Recent years have seen the development of hardware and software packages that handle auditory information on the Macintosh computer. This is good news for researchers who use auditory materials in their experiments because using these packages on the Macintosh reduces difficulty and time of preparation for these materials. The purpose of this paper is to illustrate some features of these packages by describing some experiments that benefit from using digitized sounds.

There are many hardware/software packages available for digitized sound, and although I will mention a few by name for illustrative purposes, interested researchers should check what is available and appropriate for their needs (see, e.g., a short review of available products by Heid, 1986). Two packages, MacNifty's Soundcap and Magnum's Natural Sound, each priced at around \$120, include the sound digitizer and provide excellent visual and editing manipulations of the created sound file. Other packages (e.g., G. W. Instruments' MacAdios, priced around $\$ 2500$ ) also provide powerful sound analysis features, such as spectrum analyses and plots of the fundamental frequency. In addition, many packages provide routines that allow languages such as Microsoft BASIC to read and play sound files, thereby allowing the user to program the computer to present the auditory stimuli at the rate and order needed for the experiment.

The sound signal enters the Macintosh via a sound digitizer, which is typically a small box that connects to a microphone or tape player on one end and to the Macintosh's modem or printer port on the other. Sound digitizers faithfully record a variety of sounds that might be used in an experiment, ranging from human speech to music to naturally occurring sounds. Once the sound is digitized, the software allows the user to play and edit the sound. The Macintosh's sampling resolution is about half that of compact disc equipment, but the quality of the digitized sound is still excellent. The editing features vary from product to product, but a common feature is the visual display of the sound in wave form, scaled according to time along the $x$-axis and amplitude along the $y$-axis (see

Address correspondence to Janet M. Gibson, Department of Psychology, Rice University, Houston, TX 77251.
Figure 1 for an illustration of the viewing screen of MacNifty's Soundcap sound editor). Scroll bars allow viewing of the entire sound file. Depending on the software, the sound may be scaled in seconds, 10ths of a second, or as magnified as $1 / 22200 \mathrm{sec}$. Editing the sound can be as simple as cutting and pasting, two features that are standard on software for the Macintosh. Sections of sound can be cut and pasted elsewhere within the file or can be inserted into another sound file.

One advantage of using the Macintosh for auditory stimuli is that it will often simplify the process of compiling materials. This can be illustrated by an auditory memory experiment in which, say, subjects are presented with three blocks of lists, each composed of a random ordering of the digits 1 through 9 , and 30 different lists form one block, with blocks differing in presentation rate of list items. Subjects are asked to recall the digits in serial order following each list presentation. If the experimenter were to use only a tape recorder, either the lists would have to be spoken at the correct presentation rate or the tape would have to be spliced to produce the 30 randomly ordered lists at the correct rate. On the other hand, if a sound editor on the Macintosh were used, the spoken numbers 1 through 9 could be digitized and saved as nine separate files. Then, using BASIC or some other language, the nine files could be read into memory, assembled within a matter of seconds into a random order specified by an array holding the random numbers, and played at the rate needed for that condition. This can save a lot of time. Note that this memory experiment can be run directly on the Macintosh, or each list can be taperecorded after it is assembled for later presentation on a tapeplayer during the experiment. Additional savings can be found for more complicated stimuli. For example, to study the effect of male- versus female-voiced items in the list, it is much easier to assemble lists on the computer for at least two reasons: (1) disk files do not have a temporal order, as do sounds on a tape, and femaleand male-voiced words can be digitized without regard to any future presentation order; (2) disk files have verbal labels that make it easy to tell what the file contains, thereby making it easy to select the items to be read and assembled into lists for counterbalanced designs. 


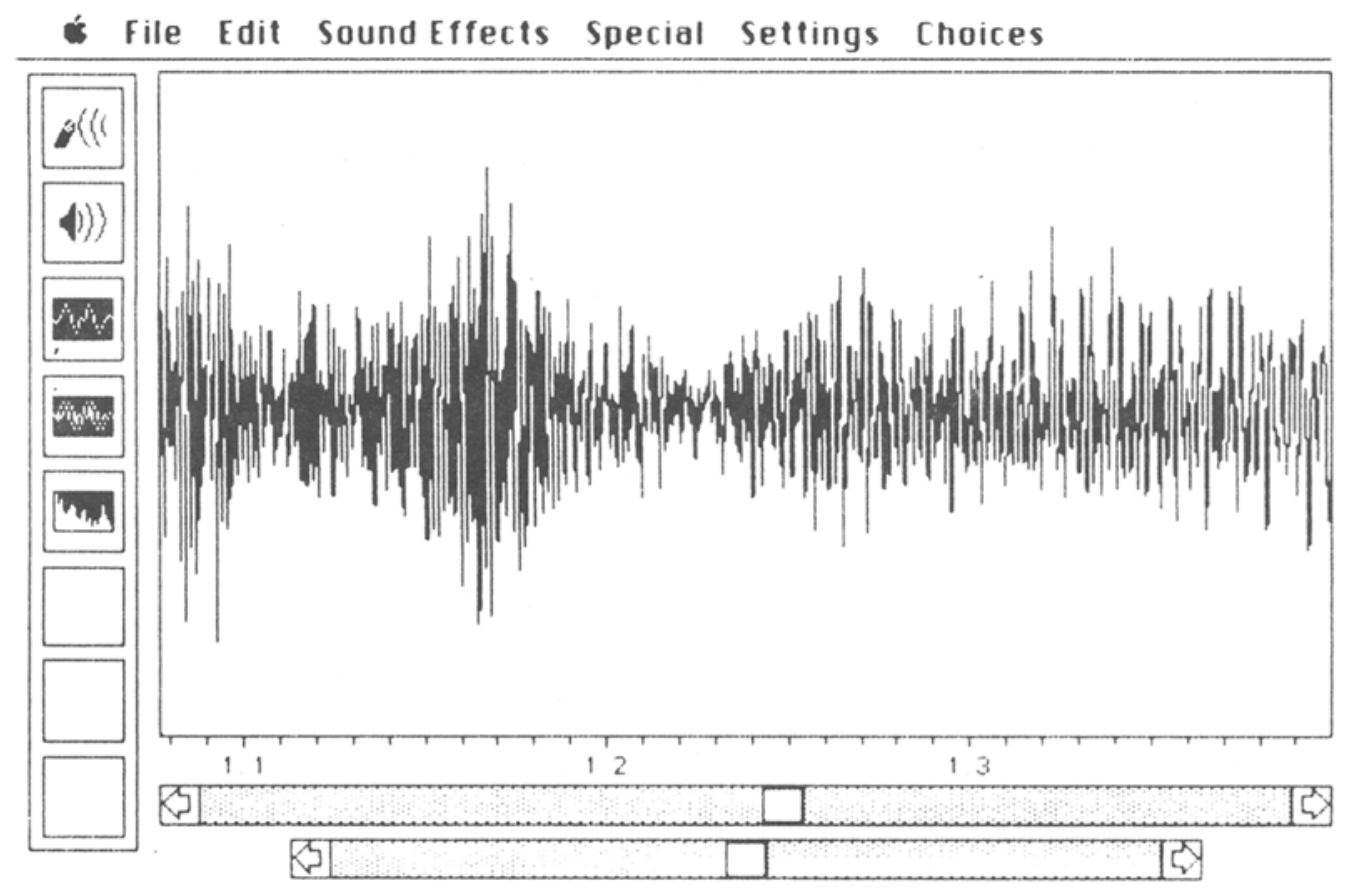

Figure 1. The editor screen of MacNifty's Soundcap. Time is scaled on the $x$-axis and amplitude on the $y$-axis.

Suppose, for another example, that an investigator wants to examine subjects' ability to detect the grammaticality of spoken sentences. A problem that may arise in preparing the material is that the speaker's intonation patterns, pauses, or stumbling over the ungrammatical sentences might well be confounded with the grammaticality variable. Sound editors on the Macintosh solve this problem by making it easy to cut and paste words within a sound file. Ungrammatical sentences can be formed by rearranging some words within a sentence, by taking a word out, or by assembling single words to form the needed sentence without messy gaps or unevenness of phrasing. All this can be done visually and with easeall that is required in most cases is a few mouse clicks and keyboard presses.

Because any section of the sound file can be edited, many types of experiments can take advantage of systematic manipulation of small differences within stimuli. For example, there is much interest in the field of cognitive psychology in how much of a word needs to be present for its recognition or identification. In one such experiment, the researcher might be interested in how much of a word must be heard in order for it to be identified when it is placed in the context of a sentence. With a sound editor, the experimenter can select how much of the word to cut according to the time scale along the $x$-axis, and it would be rather easy to vary systematically the amount cut across sentences. To give another example, experiments that have used visual presentation of word fragments to test for memory or priming can now easily be conducted with the auditory presentation of word fragments. Visual word fragments can be described as words with several blank lines replacing some letters (e.g., R__NDR_P). Auditory fragments can be made by replacing parts of the sound wave with silence, an easy option in MacNifty's Soundcap. This can be done according to the time scale (e.g., replacing every other .1 sec of sound with silence) or according to phonemes, which are usually visible in the wave. However, cutting out small portions of words can be time consuming when dealing with many words for an experiment. A language such as BASIC can be used to speed the process of replacing parts of a sound with silence. The program can create fragmented versions of a word by replacing designated elements of the array containing the sound of the word with the elements of another array containing the sound of silence. In this way, the program can work like a sound editor, but with greater speed and accuracy. ${ }^{1}$

Sound editors provide other editing features besides simple cutting and pasting. For example, sound editors allow the user to change the pitch or the volume of the original digitized sound. Sounds can also be played backward. Other features may include the capability to create an echo-like effect or make sounds fade in and out. The MacNifty Soundcap sound editor offers the option of generating white noise, which can be played alone or superimposed over another sound. Perception, attention, and memory experiments can take advantage of these sound distortion features.

Digitized versions of naturally occurring sounds (e.g., a fire engine siren) can be purchased or made from soundeffects recordings, and the sound editors can modify these sounds to meet the criteria needed for a particular experiment. For example, if $10 \mathrm{sec}$ of a bell ringing is required, 
and the sound file contains only $1 \mathrm{sec}$, it is quite easy to copy and paste the sound to get a bell ringing for the continuous $10 \mathrm{sec}$. Another valuable feature is the bent toward musical sound that these sound products possess. Digidesign's Sound Designer is especially geared to handle the sounds of musical instruments. Studies researching memory for music, recognition of melodies, or the effect of listening to different types of music while performing cognitive activities would benefit from the ability of these packages to generate or mix musical sounds with studio-like quality.

Finally, software that can analyze the digitized sound can be extremely useful for preparing stimuli for psycholinguistic experiments that often require specific sounds critical to a hypothesis. Also, this software can be used to analyze subjects' responses if the researcher is interested in subjects' speech production. Note that the Macintosh and its printer, the Imagewriter, are ideally suited for constructing and printing the graphs and pictures resulting from sound analyses.

There is one major drawback to sound editing on the Macintosh: Sound files require a lot of space, both in memory and on a disk. Sound packages vary in how much sound can be digitized at one time. On a 512K Macintosh, MacNifty's Soundcap can digitize only $15 \mathrm{sec}$ of sound at one time, while Magnum's Natural Sound and G. W. Instruments' MacAdios allow the user to digitize a sound as long as there is space on the disk to hold it. The software may allow the user to reduce the space that a file requires by varying the quality of the sound at input, so that a lower quality sound will contain less information and hence take less space. Additionally, the software may save files in a compressed form that takes less disk space than noncompressed files. For example, $1 \mathrm{sec}$ of speech digitized by MacNifty or Magnum at the highest level of input requires approximately $22 \mathrm{~K}$ in a noncompressed mode and $11 \mathrm{~K}$ in a compressed mode (for the second highest input quality, which is still good enough for speech, these numbers are reduced by half). Of course, the problem of disk space is less severe if a hard disk, rather than a singlesided $400 \mathrm{~K}$ disk, is used to hold the sound files.

Use of a program to play the sound may also limit the amount of sound that can be played at one time. Sounds are placed in arrays, which occupy RAM. The number of sound arrays that can be held in memory at one time is therefore limited to the available RAM. A onedimensional double-precision array in BASIC, which can hold at most 32,767 elements, fills $250 \mathrm{~K}$ of RAM. In terms of playing time, this is $15 \mathrm{sec}$ of continuous sound. A 512K Macintosh cannot hold much more than this array (because the program also takes up RAM); a Macintosh Plus, with its megabyte of RAM, can hold a twodimensional double-precision array of sound, but even this array is limited to under a minute's worth. Similarly, the number of smaller sound arrays held in memory at one time is limited. Because a continuous presentation of sound is restricted to the available RAM, once the limit is reached, the array(s) must be refilled before more sound can be played. Pauses in the presentation to refill arrays may or may not be a problem. For example, while the arrays are being filled with the items for the next list presentation, subjects could be writing responses to the previous list. However, if the experimenter wanted to present 3 min of continuous sound, the pauses to refill would definitely be a problem. In an experiment that requires a long continuous playing time, the experimenter would have to record the sounds on tape and use a tape player to present the stimuli. Even so, the Macintosh could be used to view, modify, or order the digitized sounds put on the tape. Future Macintoshes will have larger RAMs, which will alleviate some space limitations.

To summarize, sound packages for the Macintosh provide powerful services for using auditory stimuli in research. They allow excellent reproduction of sounds through a digitizer, and the stored sounds can be played over and over without loss of quality. Speech or other sounds can be viewed, easily manipulated, and stored with a filename for easy retrieval. The digitized sounds are less mysterious than sounds recorded on tape. The researcher does not have to rely on his or her perception of the sound, but can see the soundwave plotted against time and know its duration, pitch, and volume. Because the computer can be used to present auditory material directly in the experiment via a custom-written program, researchers can make use of the Macintosh's precise timing of presentation or its capability to record subjects' reaction times following presentations. BASIC programs can use the SPART routine in PsychLib (see Lane \& Ashby, 1987) to rapidly present visual and auditory materials together. In addition, further hardware and software development will enhance the already sophisticated sound editing and sound analysis capabilities available with contemporary packages. This offers the potential for better and more creative experiments that use auditory stimuli.

\section{REFERENCES}

HeID, J. (1986, August). Is it live or is it Mac? MacWorld, pp. 124-127. LANe, D., \& Ashry, A. (1987). PsychLib: A library of machinelanguage routines for controlling psychology experiments on the Apple Macintosh computer. Behavior Research Methods, Instruments, \& Computers, 19, 246-248.

\section{NOTE}

1. I thank Cary Jensen for pointing this out to me when I was starting to make fragments of 120 words. With the MacNifty Soundcap editor, it took me approximately 10 min to make five different fragments of one word-each fragment varied in the percentage of sound replaced with silence every .1 sec. After writing the BASIC program, I had the finished tape of all fragments of all 120 words in $2 \mathrm{~h}$. 